\title{
Application of Network Simulation in Evaluation of Network Performance Index
}

\author{
Luo Qiqiang ${ }^{1,}$, Yin Xiaoqin ${ }^{2}$ \\ ${ }^{1}$ Nanchang Institute of Science and Technology, Nanchang 330108,China \\ ${ }^{2}$ Jiangxi Tellhow Animation Vocational College, Nanchang 330020, China \\ a luoqiqiang@126.com
}

Keywords: Network simulation; virtual network platform; network performance index; network communication

\begin{abstract}
Through the network simulation software to build a virtual network platform in the computer, to simulate the real network environment, network technology development personnel can not only for network communication, network equipment, agreement on this platform, the design and research of network application, analyze and evaluate the performance of the network can. NS-3 is a discrete event network simulation driver. The operator can edit the network topology and network environment that he needs, simulate the data transmission of a network, and output its performance parameters. Network simulation technology is a new method of network planning and design. With its unique technology, the technology becomes a powerful tool for network design, which is economically, effectively and irreplaceable by other traditional methods.
\end{abstract}

\section{Introduction}

With the rapid development of computer network technology today, network researchers need to develop a new network protocol, do pioneering research for the development of the Internet; research on how to use the existing network resources to design, make the design of the network to achieve the highest performance. No matter what, all need to analyze and evaluate the network through the network. The simulation can precisely simulate the behavior of the component, to obtain sufficient data to accurately predict the performance of the system.

NS2 (Network Simulator, version is a free open source network simulation software.NS2 simulator development Lawrence California Berkeley national laboratories and other units is very powerful, strong expansibility, high efficiency, has been widely used in LAN, Wan, simulation of wireless mobile network and satellite network [1].

The rapid development of modern communication network, the network structure and the increasingly large scale, the Internet business has become increasingly rich, how to minimize the cost of the formation of excellent performance of network, and quickly and accurately from a variety of network construction scheme to select the optimal one, through the formation of the actual network test is not economical when the network is huge when it is not possible, the simulation software is used in an economical, effective and feasible method. Through the simulation, not only can optimize the design and planning of the existing network, can also carry out the research of network equipment and network protocol, through the experimental teaching, so that students can more deeply understand the application the method of OPNET software and the core technology of network engineering.

The experimental teaching task: to master the basic use of the mainstream network simulation software OPNET, including the simulation process simulation environment, grasp the simulation technology of LAN, and join the relevant service in the simulation environment. Leading the students to start OPNET, for students to in-depth study and lay a good foundation.

The physical layer (Physical Layer): the main definitions of physical equipment standards, such as the interface type interface type cable, optical fiber, various transmission medium transmission rate. Its main function is to transmit bit stream (by 1, 0 into the current intensity of transmission, arrive at 
the destination and then converted to 1,0 , that is we often said that the DAC and ADC). This layer is called the data bits.

Data link layer (Data Link Layer): defines how to format data for transmission, and how to control access to physical media. This layer usually provides error detection and correction to ensure reliable transmission of data.

Network layer (Network Layer): the connection and path selection between two host systems located in different geographical locations.Internet makes the number of users accessing the information from all the world sites greatly increased, and the network layer is the layer to manage this connection.

At present, the planning and design of the data network is generally used in experience, network design and calculation of the traditional test method. However, when the network scale is more and more big, the element type is increasing, the network topology is becoming increasingly complex, network traffic is complicated, the drawbacks of network design method based on experience is more and more apparent [2]. Design experience is relatively lack of large network, so in the design process of the subjective element is more prominent.

Mathematical calculation and estimation methods for large complex networks is often very difficult, the credibility of the results is relatively low, especially for packet switched data network, statistical multiplexing, the situation is more serious. Therefore, with the continuous expansion of the network, the reliability is more and more need for a new network planning and the design methods to improve the network design objective and design results, reducing the risk of investment in network construction. The network simulation technology came into being in this demand. The network simulation technology with a particular method, which is capable for network planning and design to provide objective and reliable quantitative basis, shorten the period of network construction, improve the scientific decision making in network construction, reduce the risk of investment in network construction.

\section{Literature review of network simulation technology}

NS2 (Network Simulator version 2), NS (Network Simulator) is a network technology based on open source code, free software simulation platform [3]. The computer network is a complex system, including a variety of communication protocols and network technology, network simulation is one of the important means of network communication technology, network refers to the use of computer simulation software on the network protocol, network topology, a research method to simulate and analyze the performance of it.

NS2 network is a kind of object-oriented network simulator is a discrete event simulator, which can simulate all kinds of different IP network, the realization of some network protocols, such as TCP and UDP, but also includes the business the source traffic generator, such as FTP, CBR and.NS2 using $\mathrm{C}++$ and Otcl as the development language.NS can be said to be Otcl script interpreter, which contains the simulation event scheduler, network component object library The network construction and the model base of.NS is written in Otcl and C++.

For efficiency reasons, NS will achieve data channel and control channel separation. In order to reduce processing time and packet events, the basic network component object and event scheduler data channel on the use of $\mathrm{C}++$ to write and compile these objects by mapping. The Otcl interpreter is visible. When the simulation is completed, NS will produce one or more trace files based on text. Just add some simple statements in the Tcl script, the file will contain detailed tracking information. These data can be used to analyze the next step, you can also use NAM to show the whole the simulation process.

NS-3 is a discrete event network simulation driver [4]. The operator can edit the network topology and network environment that he needs, simulate the data transmission of a network, and output its performance parameters.

There are many modules in the software: the node module (the creation node), the mobile module (the simulation WIFI, the LTE can be used), 
Random modules (generating random error models), network modules (different communication protocols), application modules (create packet packets and accept packet packets), statistical modules (output statistics, network performance parameters) and so on. First, suppose a simple network topology: two nodes use point to point links and use TCP protocol to communicate, assuming that the random error rate is 0.00001 , and nodes are not mobile (because not wireless networks) [5].

From the aspect of matching protocol and routing choice, due to the limitation of sensor node itself and the application demand, can not fully use the 7 layer network protocol standard or TCP/IP protocol. In order to extend the network lifetime, often need to redefine the network protocol, especially in routing and transmission protocol design to a key role in reducing energy consumption, the shortest path therefore requires the selection of data transmission. But the shortest path selection is a NP hard problem, especially the need to weigh in extending the network lifetime and the overall data transmission in the shortest path.

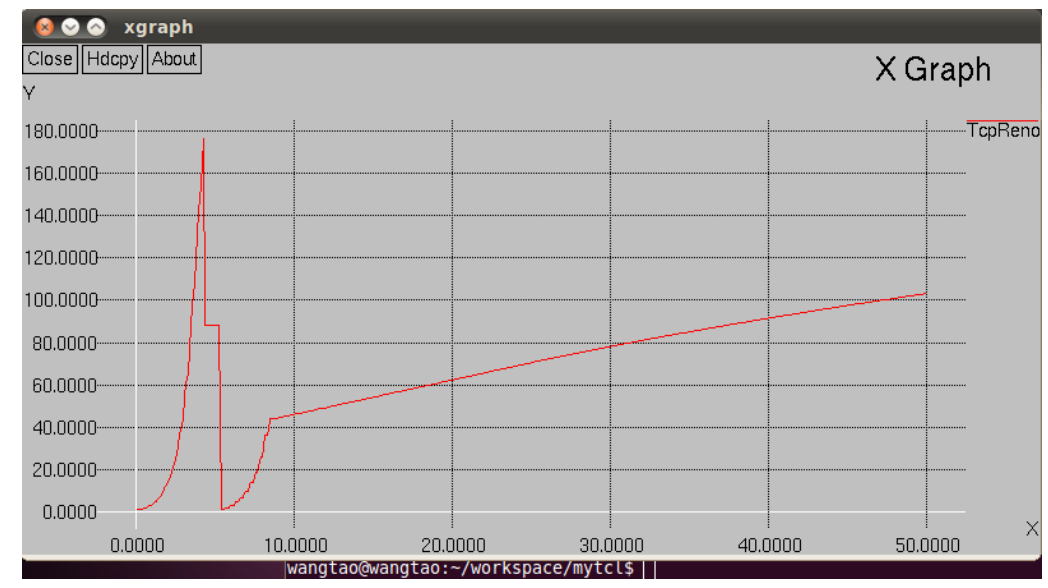

Figure 1. NAM to show the whole the simulation process

The application of using the principle of network communication are the same, but there can be a variety of ways. The damage is very small in the network development environment, the communication process of the impact on application performance is not significant, developers tend to ignore the optimization for the communication process, but that is the most probably it did not actually happen protocol.

The different impact on the performance of the process will not damage the larger network greatly, however, tend to amplify the effects of communication process on application performance. If the process design is not reasonable, resulting in the network damage effect of multiple stacking, will seriously affect the application performance, can not even use [6].

A simple example: a ERP the client software and server interaction process in the beginning of the design stage in order to obtain correct information, a large packet of information is split into a plurality of small packet transmission Every time, need to confirm the end. In the development environment, the system works well, but when the system is running in the SHUNRA simulator simulation network, client response speed slow, when SHUNRA finally found some real network simulator simulation about $100 \mathrm{~ms}$ delay characteristics, the communication process of the system seriously slow down the whole system the pros and cons of speed. Comprehensive evaluation of all aspects, after modifying the ERP communication process, the problem can be alleviated. To avoid the real deployment to the problems caused losses after the actual network.

\section{Network performance simulation NS2 and NS3 design}

The transport layer (Transport Layer): defines the data transmission protocol and port (WWW port 80), such as: TCP (transmission control protocol, low transmission efficiency, high reliability, high reliability requirements for the transmission of a large amount of data, data), UDP (user datagram protocol, and TCP characteristics on the contrary for the transmission, reliability is not high, the small amount of data data, such as QQ chat data is transmitted in this way). The main is segmented from the 
data receiving and transmission layer, to the destination address after the reorganization. Often this layer is called data segment [7].

The session layer (Session Layer): the transport layer (port number: transmission port and the receiving port access) establish data transmission. To initiate a conversation between your main system or accept session request (equipment between the need to know each other can IP it can be MAC or host name).

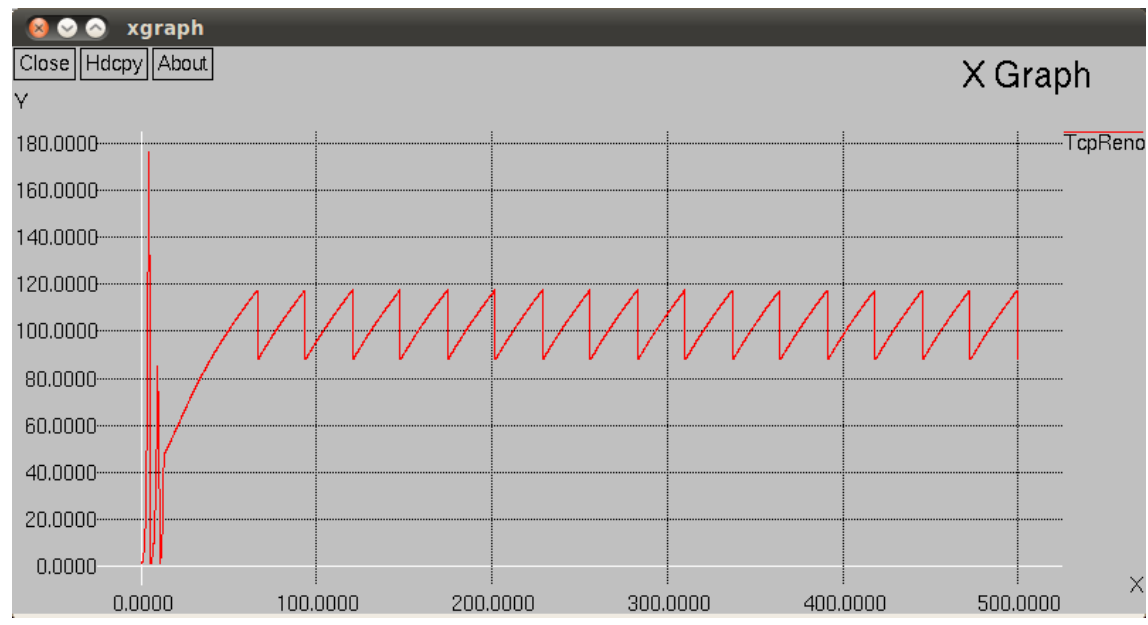

Figure 2. The transport layer (port number: transmission port and the receiving port access) establish data transmission

The layer (Presentation Layer) can ensure that sent by the application layer of a system of application layer information can be another system to read. For example, PC communication program and another computer, one of the computers using the extended 21 decimal interchange code (EBCDIC), and the other one is the use of the United States Standard Code for information interchange (ASCII) to represent the same character. If necessary, the presentation layer through the use of a general format to achieve data conversion.

Network simulation technology is a statistical model of network equipment and network link, and simulates the transmission of network traffic. Therefore, the simulation technology is based on the statistical model rather than the mathematical calculation, because the simulation is based on the network performance data needed for network design or optimization. The randomness of statistical reuse is precisely reproduced. It provides objective and reliable quantitative basis for network planning and design by its unique method, shortens the period of network construction and improves the scientific decision-making in network construction. Reduce the investment risk of network construction.

Network simulation software through the establishment of a virtual network platform on the computer to achieve the simulation of the real network environment, network technology developers on this platform can not only network communications, network equipment, protocols. And the network application design research also can analyze and evaluate the network performance. In addition, the simulation software provided by the simulation operation and results analysis functions to enable developers too quickly. Get the network performance parameters intuitively, provide a more convenient and effective means for optimizing design or making decisions. So use network simulation software to network protocol. Algorithm simulation has become an essential part of computer network communication research.

GENESIS is an open source tool. GENESIS software supports its own script language and script files. It implements.GENESIS in an interpreted way. It has strong scalability and provides an interface to add new commands and functions, so that users can easily add new concepts and verify them. 


\section{Application of network simulation in evaluation of network performance index}

A direct association between the simplest ways to design the central node is packet flow and peripheral node in the center of the output section of the address. Another method is to keep a destination address and transmitter cable conversion between the center node in drainage model. In this model, more suitable for the destination address and cable direct mapping drainage between.

Here, the central node model is designed as a process module consisting of four point-to-point transceiver / transceiver pairs and a relay module used to relay function packets from transceiver to transmitter. As shown in Figure 3.

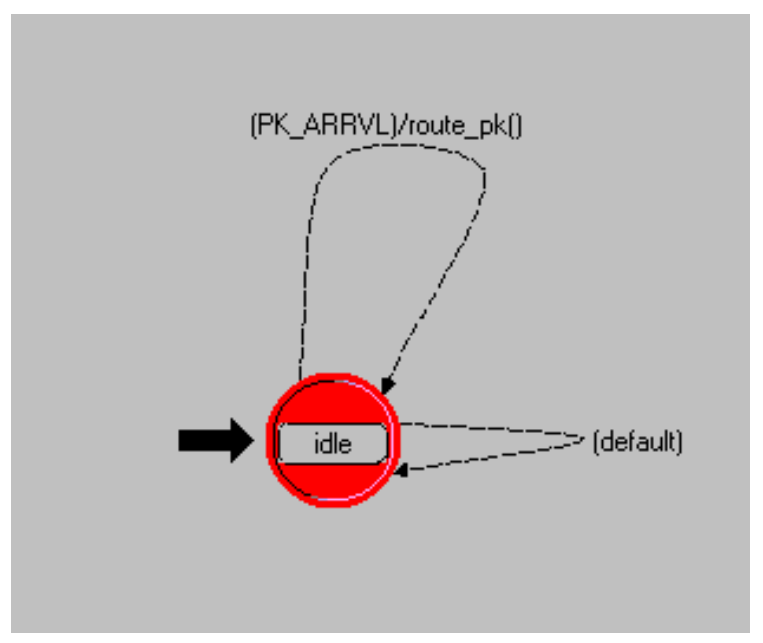

Figure 3. relay function packets from transceiver to transmitter

At present, program development is highly modular, many systems integrated a lot of outsourced code modules, if some of the modules involved in network communication. There is no doubt that we also need to verify its effectiveness and performance. We use SHUNRA simulator to provide simulation network environment and test it before integrating outsourcing modules into the system. A clearer understanding of its function and performance will reduce the risk of not knowing the performance of the outsourced module and provide a reference for the overall performance evaluation of the system.

The SHUNRA simulator provides the most realistic simulation environment for testers, which enables users to test the performance of application systems in various network environments at very low cost in the short term.

At present, no matter how big or small the application system is, it is very rare to develop and use it without testing. The more the test environment is, the closer it is to the real use environment. But how to build a test environment close to the real environment, is a very troublesome problem. For the network topology, we can refer to the real network key equipment to build the test network. It makes the test network structure similar to the real network, but there are more things can not be imitated: packet loss, delay, jitter, repetition, link interruption and congestion in the real network damage. How do we simulate their existence? It should be emphasized that it is the damage that causes the poor performance of the real system. If only the structure of the real network is built. Without trying to reproduce the network damage that also exists on the actual network. Then the test results can only reflect the function of the tested system from a limited aspect, but the incomplete test environment can not reflect the function and performance of the tested system on the actual network.

\section{Summary}

NS2 is an object-oriented network simulator. On the whole NS2 can be divided into three parts, the two part is provided to the user interface, the other part is the core of the emulator. The user interface is composed of Tcl/Tk 1, OTcl extended the interpreter, the user through the use of TCL/TK, OTcl scripting language, written in network simulation script file. Scripting language concise, intuitive, 
user without much understanding of the internal mechanism of the core simulator can use the NS2. core simulator NS2 using $\mathrm{C}++$ code written by. Tclcl $\mathrm{C}++$, the mechanism of object oriented provides NS2 and Tcl/Tk, the connection of the OTcl interpreter. User interface 2 refers to the NAM, such as Xgraph graphics tools the simulator, executing the simulation script file prepared by the user, the operation results recorded, then record the documents by the NAM program and visual display.

\section{References}

[1] Xu Leiming.NS2 and network simulation. Beijing: people post and telecommunications, 2013.6.

[2] Carl Timm, Wade Edwards. CCNP building scalable cisco internetworks study guide642-801. Beijing: Publishing House of Electronics Industry 2014:59.

[3] Lei Qing, Wang Xinggang, computer network simulation methods and tools, Journal of Communications, 2011 9: 84-90.

[4] Reajie R,Handley M,Estin D. RAP:An end—to-end rates-based congestion control mechanism for real time streams in the intemet. In:IEEE INFOCOM2014. New York, 2015:1337-1345.

[5] Lu Zhengfu, Liu Jiqing, Network performance Simulation experiment based on OPNETModeler, Experimental Science and Technology,2016.

[6] L.Zhao, C.Fan.Enhancement of QoS differentiation OVer IEEE 802.11 WLAN, IEEE Commun.Lett. 2014, (8):492-496.

[7] Hou Zonghao, Wang Bingkang, Huang Yongxiang, research on network simulation. Computer simulation, 2014, 20 (10). 